\title{
Adherence to the Mediterranean diet is associated with better mental and physical health
}

\author{
Miguel-Angel Muñoz ${ }^{1,2}$, Montserrat Fíto ${ }^{1,3}$, Jaume Marrugat ${ }^{4,5,6}$, Maria-Isabel Covas ${ }^{1,3}$, \\ Helmut Schröder ${ }^{1,3 *}$ and on behalf of the REGICOR and HERMES investigators $\dagger$ \\ ${ }^{1}$ Cardiovascular Risk and Nutrition Research Group (CARIN-ULEC), IMIM-Hospital del Mar Barcelona, Biomedical Research \\ Park (Parc de Recerca Biomèdica de Barcelona - PRBB), c/Doctor Aiguader 88, 08003 Barcelona, Spain \\ ${ }^{2}$ Equipo de Atención Primaria Montornés-Montmeló, Institut Català de la Salut, Pza Ernest Lluch 1, 08160 Montmeto (Barcelona), Spain \\ ${ }^{3}$ Centros de Investigación Biomédica en Red (CIBER), Fisiopatología de la Obesidad y Nutrición (CIBEROBN), Instituto de Salud \\ Carlos III, c/Sinesio Delgado 6, 28029 Madrid, Madrid, Spain \\ ${ }^{4}$ Cardiovascular Epidemiology and Genetics Research Group (EGEC-ULEC), IMIM-Hospital del Mar, c/Doctor Aiguader 88, \\ 08003 Barcelona, Spain \\ ${ }^{5}$ Program of Research in Inflammatory and Cardiovascular Disorders (RICAD), IMIM-Hospital del Mar, c/Doctor Aiguader 88, \\ 08003, Barcelona, Spain \\ ${ }^{6}$ Centros de Investigación Biomédica en Red (CIBER), Epidemiología y Salud Pública (CIBERESP), Instituto de Salud Carlos III, \\ c/Sinesio Delgado 6, 28029 Madrid, Madrid, Spain
}

(Received 11 April 2008 - Revised 26 September 2008 - Accepted 29 September 2008 - First published online 15 December 2008$)$

\begin{abstract}
The aim of the present study was to analyse the association between adherence to the Mediterranean diet and self-perceived mental and physical health function, controlled for confounding effects of age, smoking, BMI, alcohol consumption, educational level, leisure-time physical activity and the presence of chronic conditions. A random sample of the 35-74-year-old population (3910 men and 4285 women) of Gerona, Spain, was examined in 2000 and 2005 in two independent population-based cross-sectional surveys. Dietary intake was assessed using a validated FFQ. The Mediterranean diet score (MDS) was calculated according to tertile distribution of energy-adjusted food consumption considered characteristic for the Mediterranean region. Health-related quality of life was measured using the SF-12 questionnaire. Alcohol consumption, leisure-time physical activity and smoking habits were recorded. Weight and height were measured. Age-adjusted linear regression analysis revealed a significant $(P<0.01)$ direct association of the MDS with self-reported mental and physical health in both sexes. An increase of 5 units of the MDS was directly associated with changes of 0.74 and 1.15 units in men and women, respectively, in the mental component score after controlling for potential confounders. The age-adjusted direct association of the MDS with self-reported scoring of physical health remained stable after adjusting for several confounders in men but was attenuated in women. Adherence to the Mediterranean diet was associated with higher scoring for self-perceived health.
\end{abstract}

Mental health: Mediterranean diet: Diet quality

The traditional dietary pattern of Mediterranean populations offers a palatable choice of healthy eating. This diet is generally characterized, despite regional disparities, by high consumption of plant foods, moderate consumption of alcohol and low consumption of meat. There is consistent evidence for the health-promoting properties of the Mediterranean $\operatorname{diet}^{(1)}$. Individual components of this diet can partially explain the health-protecting effects ${ }^{(2)}$.

Analysis of quality of life is a valid measurement for self-perceived mental and physical health status, which is closely associated with CVD and all-cause mortality ${ }^{(3,4)}$. It has been argued that the social and cultural aspects related to the Mediterranean diet may additionally provide health benefits ${ }^{(5)}$. A favourable environment and social support may contribute to having a better self-perceived health-related quality of life (HRQL) ${ }^{(6)}$. Furthermore, there is evidence for the association of self-perceived health status and diet. Poor diet quality, characterized through low consumption of particular healthy foods or nutrients, has been associated with low mental and physical health $^{(7-10)}$. Furthermore, evidence from dietary intervention studies indicates an improvement of quality of life through healthy diets ${ }^{(11,12)}$. However, to our knowledge it is unknown if healthy diet, such as the Mediterranean diet, affects selfperception of HRQL in the general population. The aim of the

Abbreviations: HRQL, health-related quality of life; MCS, mental component score; MDS, Mediterranean diet score; PCS, physical component score.

* Corresponding author: Dr Helmut Schröder, CARIN-ULEC, fax +34933160 796, email hschroeder@imim.es

$\dagger$ A full roster of REGICOR investigators and collaborators can be found at email www.REGICOR.org/regicor.inv 
present study was to analyse the association between adherence to the Mediterranean diet and self-perceived mental and physical health, controlled for confounding effects of age, smoking, BMI, alcohol consumption, educational level, leisure-time physical activity and the presence of chronic conditions in a representative population of Spanish men and women.

\section{Methods}

\section{Study participants}

Data were obtained from two population-based cross-sectional surveys conducted in Gerona (Spain) in 2000 and 2005. The first survey included 3054 randomly selected free-living men and women between 25 and 74 years of age. The second survey included 6322 men and women between 35 and 88 years of age. Response rates for the two surveys were 71.0 and $71.5 \%$, respectively. Participants from both surveys aged 35-74 years ( $n$ 8195) were included in the present analysis. Participants with unrealistic dietary energy intake ( $n$ 420), defined as energy intake less than $3347 \mathrm{~kJ} / \mathrm{d}(800 \mathrm{kcal} / \mathrm{d})$ or more than $18828 \mathrm{~kJ} / \mathrm{d}$ $(4500 \mathrm{kcal} / \mathrm{d})$, were excluded from analysis. Furthermore, data on self-reported mental and physical health function were missing for 650 participants. Finally, 7145 men and women (87.2\%) were included in the analysis. The protocol was approved by an Ethics Committee (CEIC-IMAS, Barcelona, Spain) and the results of the examination were sent to all participants.

\section{Quality of life}

We chose one of the most widely used generic tests in health outcomes research to analyse self-reported HRQL. The SF-12 Health Survey was developed to provide a shorter alternative to the MOS SF-36 Health Survey ${ }^{(13)}$ to measure overall physical and mental health outcomes. The SF-12 is a valid measurement tool for the exploration of self-perceived vitality, pain, mental health, physical, social, emotional and mental functioning, and general health; it has been validated for use in the Spanish population ${ }^{(14)}$. This test consists of twelve questions addressing eight items that are summarized in a physical health score (items: physical functioning, role - physical, bodily pain, general health), and a mental health score (items: vitality, social functioning, role - emotional, mental health). The questionnaire uses Likert scales to analyse the intensity or frequency of each response. The outcomes are standardized according to the population-based values, considering 50 (SD 10) as the mean of the population. In Spain, mean values of physical and mental health scores are 49.9 (SD 9.0) and 51.0 (SD 9.0), respectively ${ }^{(14)}$.

\section{Dietary assessment}

Food consumption and nutrient intake were measured by a validated $\mathrm{FFQ}^{(15)}$ administered by a trained interviewer. An optical readable FFQ asked for the usual intake over the past year. In a 165-item food list, including alcoholic and non-alcoholic beverages, the participants indicated their usual consumption and chose one of ten frequency categories, from never or less than once per month to six or more times per day. Instead of standard questions about portion size, this FFQ uses medium servings defined by natural (e.g. one orange, one standard slice) or households units (e.g. one cup, one spoon).
A Mediterranean diet score (MDS) based on the intake of cereals, vegetables, fruits, legumes, nuts, fish, dairy products, olive oil, meat and red wine was computed to calculate overall diet quality. Multiple food components were operationalized as a single dietary exposure variable, similar to the approach used by other authors ${ }^{(16-18)}$. The characteristics of the scoring system are based on the traditional food consumption of the Mediterranean region ${ }^{(16)}$. With the exception of red wine, the MDS was calculated according to the tertile distribution of energy-adjusted ${ }^{(19)}$ consumption of cereals, vegetables, fruits, legumes, nuts, fish, dairy products, olive oil, meat and red wine. The lowest tertile was coded as 1 , medium as 2 and highest as 3 for cereals, fruits, vegetables, legumes, fish, olive oil and nuts. The highest tertile was coded as 1, medium as 2 and lowest as 3 for meat and dairy products. Moderate red wine consumption was included as a favourable component in the MDS. For this purpose we coded up to $20 \mathrm{~g}$ alcohol intake proceeding from red wine as 3. Exceeding this upper limit or reporting no red wine consumption was coded with 0 . The distribution values of all dietary components were calculated. The resulting MDS ranged from 10 to 30 .

\section{Measurement of non-dietary variables}

Information on demographic and socio-economic variables, medical history, diet and lifestyle factors including tobacco smoking and alcohol consumption was obtained through structured standard questionnaires administered by trained personnel.

Leisure-time physical activity was measured by the Minnesota leisure-time physical activity questionnaire, also administered by a trained interviewer. This questionnaire has been previously validated for Spanish men and women ${ }^{(20,21)}$.

\section{Anthropometric data}

Measurements were performed by a team of trained nurses and interviewers who used the same standard methods in the two surveys. A precision scale of easy calibration was used for weight measurement with participants in underwear. Body weight was rounded up to $200 \mathrm{~g}$ and height was measured to the nearest $0.5 \mathrm{~cm}$. BMI was calculated as weight/height squared $\left(\mathrm{kg} / \mathrm{m}^{2}\right)$.

\section{Statistical analyses}

Differences in continuous variables were compared using the Student $t$ test. The Mann-Whitney $U$ test was used for nonparametric variables. Categorical variables were tested using the $\chi^{2}$ goodness of fit test. The general linear modelling procedures (PROC GLM version 8.0; SAS Institute, Cary, NC, USA) were used to estimate lifestyle, anthropometric, medical history and socioeconomic variables according to the tertile distribution of the SF-12 mental component score (MCS) and physical component score (PCS). Linear trend was tested through including the categorized variable (tertile distribution of the SF-12 MCS and PCS) as continuous in this model. The polynomial contrast was used to determine $P$ for linear trend for continuous variables. Age-adjusted logistic regression was performed to calculate $P$ for linear trend of dichotomous variables (educational level, current smokers, 
dyslipidaemia, diabetes and hypertension) according to tertile distribution of physical and mental health. Dichotomous variables were included in these models as the dependent variable and tertiles of PCS and MCS as continuous explanatory variables (PROC LOGISTIC procedure of SAS version 8.0; SAS Institute). Multiple linear regression models (PROREG version 8.0; SAS Institute) were fitted to determine the confounder-controlled association of the MDS with self-reported mental and physical health function. The data of these models followed a normal distribution. Differences were considered significant if $P<0 \cdot 05$.

\section{Results}

No significant differences were found for age $(P=0 \cdot 163)$, leisure-time physical activity $(0.460)$ and smoking status $(P=0.422)$ between subjects who furnished complete and valid information on the study variables and those who failed to do so. Participants who were excluded from analysis drank less alcohol (not selected participants 9.5 (SD 19.3) g/d v. $10 \cdot 0(\mathrm{SD} 15 \cdot 0) \mathrm{g} / \mathrm{d}$ selected participants, $P=0.001)$ and had lower education levels (not selected participants $35.5 \%$ chose 'more than primary school' $v .47 .3 \%$ of selected participants, $P<0 \cdot 001)$.

Men spent more time in leisure-time physical activity, had more education, consumed more alcohol, had higher BMI and showed higher scoring for self-reported physical and mental health than women (Table 1). Furthermore, the prevalence of smoking and medical history and/or drug treatment of hypertension, diabetes and dyslipidaemia was higher in men than women (Table 1).

The proportion of smokers decreased with higher scoring of mental health in both sexes (Table 2). Men and women showed an opposite linear trend of educational level according to scoring of self-reported mental health. The proportion of higher-educated men decreased across tertile distribution of self-reported mental health and increased in women. Alcohol consumption in men and leisure-time physical activity in women increased across tertile distribution of self-reported mental health. Women with higher scoring of self-reported mental health had a lower BMI and a lower prevalence of medical history and/or drug treatment of dyslipidaemia (Table 2).

Age, BMI and the prevalence of medical history and/or drug treatment of diabetes and hypertension decreased across tertile distribution of self-reported physical health in both sexes (Table 3). A direct association was observed between physical health and both leisure-time physical activity and the proportion of higher educational level in men and women. Higher scoring of self-reported physical health was associated with a lower prevalence of smoking and medical history in men but with higher alcohol consumption in women (Table 3).

Multiple linear regression models were fitted to analyse the association of the MDS with self-reported mental and physical health in men and women (Table 4). The age-adjusted models revealed a significant direct association of the MDS with selfreported mental and physical health in both sexes. An increase of 5 units of the MDS was directly associated with changes of 0.74 and 1.15 units in men and women, respectively, in the MCS after controlling for potential confounders. The ageadjusted direct association of the MDS with self-reported scoring of physical health remained stable after adjusting for several confounders in men but was attenuated in women. There were no significant interactions between MDS and BMI, leisure-time physical activity or smoking.

\section{Discussion}

To our knowledge this is the first study to take in a holistic approach in analysing the relationship between self-perception of HRQL and diet. In the present population, self-perceived mental and physical health was directly associated with adherence to the Mediterranean diet in men and women. This association held, with the exception of physical health function in women, after controlling for confounders.

Intervention studies lowering dietary fat have reported improvement in mood, hostility and psychological wellbeing and a decrease in depression and anxiety ${ }^{(22-26)}$. Additionally, intervention with a high-carbohydrate diet and

Table 1. Characteristics of the study participants

(Mean values, standard deviations and $95 \% \mathrm{Cl}$ )

\begin{tabular}{|c|c|c|c|c|c|}
\hline & \multicolumn{2}{|c|}{ Men ( $n$ 3448) } & \multicolumn{2}{|c|}{ Women $(n$ 3697) } & \multirow[b]{2}{*}{$P$} \\
\hline & Mean & SD or $95 \% \mathrm{Cl}$ & Mean & SD or $95 \% \mathrm{Cl}$ & \\
\hline Age (years) & $54 \cdot 3$ & $11 \cdot 0$ & $53 \cdot 8$ & $10 \cdot 9$ & 0.082 \\
\hline SF-12 PCS (units) & $51 \cdot 2$ & 7.9 & $48 \cdot 7$ & $9 \cdot 7$ & $<0.001$ \\
\hline SF-12 MCS (units) & $49 \cdot 6$ & $9 \cdot 8$ & $45 \cdot 1$ & 11.4 & $<0.001$ \\
\hline Leisure-time physical activity $(\mathrm{MET} \times \min / \mathrm{d})$ & 371.6 & $379 \cdot 8$ & $243 \cdot 6$ & $245 \cdot 2$ & $<0.001$ \\
\hline BMI $\left(\mathrm{kg} / \mathrm{m}^{2}\right)$ & $27 \cdot 8$ & $3 \cdot 8$ & $27 \cdot 1$ & $5 \cdot 1$ & $<0.001$ \\
\hline Current smoker (\%)* & 31.5 & $30 \cdot 1,32 \cdot 9$ & $16 \cdot 6$ & $15 \cdot 3,18 \cdot 0$ & $<0.001$ \\
\hline Alcohol consumption (g/d) & $15 \cdot 9$ & $18 \cdot 3$ & 4.6 & $7 \cdot 9$ & $<0.001$ \\
\hline Educational status (\%)† & $48 \cdot 6$ & $47 \cdot 0,50 \cdot 1$ & $46 \cdot 0$ & $44 \cdot 4,47 \cdot 6$ & 0.025 \\
\hline \multicolumn{6}{|l|}{ Medical history and/or drug treatment of } \\
\hline Hypertension (\%) & $32 \cdot 1$ & $30 \cdot 6,33 \cdot 7$ & $29 \cdot 1$ & $27 \cdot 6,30 \cdot 5$ & 0.005 \\
\hline Diabetes $(\%)$ & 13.5 & $12 \cdot 4,14 \cdot 6$ & $11 \cdot 3$ & $10 \cdot 3,12 \cdot 4$ & 0.005 \\
\hline Dyslipidaemia (\%) & $33 \cdot 6$ & $32 \cdot 0,35 \cdot 1$ & $30 \cdot 1$ & $28 \cdot 6,31 \cdot 6$ & 0.002 \\
\hline
\end{tabular}

MCS, mental component score; MET, metabolic equivalents; PCS, physical component score; SF-12, SF-12 Health Survey.

${ }^{*}$ Categorical variables are presented as relative frequencies.

† Higher than primary school. 
Table 2. Lifestyle, anthropometric and socio-demographic variables according to tertile distribution of self-reported mental health (SF-12 Health Survey mental component score) in men and women (adjusted for age) ${ }^{*}$

(Mean values and $95 \% \mathrm{Cl}$ )

\begin{tabular}{|c|c|c|c|c|c|c|c|c|}
\hline & \multicolumn{4}{|c|}{ Men } & \multicolumn{4}{|c|}{ Women } \\
\hline & $\begin{array}{l}\text { First tertile } \\
(7 \cdot 6-48.6)\end{array}$ & $\begin{array}{l}\text { Second tertile } \\
(48.7-55.4)\end{array}$ & $\begin{array}{l}\text { Third tertile } \\
(55.5-68.6)\end{array}$ & $\begin{array}{l}P \text { for linear } \\
\text { trend }\end{array}$ & $\begin{array}{l}\text { First tertile } \\
(5 \cdot 2-41 \cdot 7)\end{array}$ & $\begin{array}{l}\text { Second tertile } \\
(41 \cdot 8-52 \cdot 0)\end{array}$ & $\begin{array}{l}\text { Third tertile } \\
(52.1-70.9)\end{array}$ & $\begin{array}{l}P \text { for linear } \\
\text { trend }\end{array}$ \\
\hline$n$ & 1140 & 1138 & 1171 & & 1216 & 1214 & 1267 & \\
\hline Age (years) & & & & $<0.001$ & & & & 0.285 \\
\hline Mean & $53 \cdot 2$ & $52 \cdot 6$ & $56 \cdot 3$ & & 54.5 & $52 \cdot 8$ & $54 \cdot 1$ & \\
\hline $95 \% \mathrm{Cl}$ & $53 \cdot 2,54 \cdot 5$ & $51 \cdot 9,53 \cdot 2$ & $55 \cdot 7,56 \cdot 9$ & & $53 \cdot 9,55 \cdot 1$ & $52 \cdot 2,53 \cdot 4$ & $53 \cdot 4,54 \cdot 7$ & \\
\hline Current smokers & & & & 0.031 & & & & 0.009 \\
\hline$\%$ & 34.2 & 30.4 & $30 \cdot 0$ & & $18 \cdot 9$ & $16 \cdot 0$ & $15 \cdot 1$ & \\
\hline $95 \% \mathrm{Cl}$ & $31.5,36.9$ & $27 \cdot 7,33 \cdot 0$ & $27 \cdot 4,32 \cdot 7$ & & $16 \cdot 9,20 \cdot 9$ & $14 \cdot 0,18 \cdot 0$ & $13 \cdot 1,17 \cdot 0$ & \\
\hline Alcohol consumption $(\mathrm{g} / \mathrm{d})$ & & & & 0.165 & & & & 0.029 \\
\hline Mean & $15 \cdot 5$ & $15 \cdot 5$ & $16 \cdot 6$ & & 4.3 & 4.4 & 5.0 & \\
\hline $95 \% \mathrm{Cl}$ & $14 \cdot 4,16 \cdot 6$ & $14 \cdot 4,16 \cdot 5$ & $15 \cdot 5,17 \cdot 6$ & & $2 \cdot 8,4 \cdot 7$ & $3.9,4.8$ & $4.5 ; 5 \cdot 4$ & \\
\hline LTPA $($ MET $\times \min / d)$ & & & & 0.001 & & & & 0.124 \\
\hline Mean & 347 & 365 & 399 & & 234 & 248 & 249 & \\
\hline $95 \% \mathrm{Cl}$ & 325,369 & 343,389 & 377,420 & & 220,248 & 234,261 & 236,262 & \\
\hline BMI $\left(\mathrm{kg} / \mathrm{m}^{2}\right)$ & & & & 0.312 & & & & 0.007 \\
\hline Mean & $27 \cdot 8$ & $27 \cdot 7$ & $28 \cdot 0$ & & $27 \cdot 6$ & $26 \cdot 7$ & $27 \cdot 1$ & \\
\hline $95 \% \mathrm{Cl}$ & $27 \cdot 6,28 \cdot 1$ & $27 \cdot 5,27 \cdot 9$ & $27 \cdot 8,28 \cdot 2$ & & $27 \cdot 4,27 \cdot 9$ & $26 \cdot 4,27 \cdot 0$ & $26 \cdot 8,27 \cdot 3$ & \\
\hline Educational level† & & & & 0.008 & & & & 0.001 \\
\hline$\%$ & $49 \cdot 2$ & $53 \cdot 1$ & $43 \cdot 8$ & & $42 \cdot 3$ & $47 \cdot 4$ & $48 \cdot 2$ & \\
\hline $95 \% \mathrm{Cl}$ & $46 \cdot 3,52 \cdot 0$ & $50 \cdot 3,55 \cdot 9$ & $41 \cdot 0,46 \cdot 8$ & & $39 \cdot 7,44.9$ & $44 \cdot 8,50 \cdot 0$ & $45 \cdot 6,50 \cdot 7$ & \\
\hline \multicolumn{9}{|l|}{$\begin{array}{l}\text { Drug treatment and/or } \\
\text { medical history of } \\
\text { Dyslipidaemia }\end{array}$} \\
\hline$\%$ & $35 \cdot 2$ & 31.4 & $34 \cdot 1$ & & 33.0 & $28 \cdot 7$ & $28 \cdot 7$ & \\
\hline $95 \% \mathrm{Cl}$ & $32 \cdot 5,38 \cdot 0$ & $28 \cdot 6,34 \cdot 1$ & $31 \cdot 4,36 \cdot 8$ & 0.561 & $30.5,35.5$ & $26 \cdot 2,32 \cdot 1$ & $26 \cdot 2,31 \cdot 1$ & 0.017 \\
\hline \multicolumn{9}{|l|}{ Diabetes } \\
\hline$\%$ & 13.9 & $12 \cdot 9$ & 13.5 & & $12 \cdot 7$ & $10 \cdot 0$ & 11.4 & \\
\hline $95 \% \mathrm{Cl}$ & $11 \cdot 9,15 \cdot 8$ & $11 \cdot 0,14.9$ & $11 \cdot 6,15 \cdot 5$ & 0.820 & $10 \cdot 9,14 \cdot 4$ & $8 \cdot 2,11 \cdot 8$ & $9 \cdot 6,13 \cdot 3$ & 0.329 \\
\hline \multicolumn{9}{|l|}{ Hypertension } \\
\hline$\%$ & 34.4 & 31.3 & $30 \cdot 7$ & 0.055 & 29.5 & 29.6 & 28.1 & 0.454 \\
\hline $95 \% \mathrm{Cl}$ & $31 \cdot 8,37 \cdot 0$ & $28.6,33.9$ & $28 \cdot 1,33 \cdot 3$ & & $27 \cdot 1,31 \cdot 9$ & $27 \cdot 2,32 \cdot 0$ & $25 \cdot 8,30 \cdot 5$ & \\
\hline
\end{tabular}

LTPA, leisure-time physical activity; MET, metabolic equivalents.

${ }^{*}$ For details of subjects and procedures, see Methods.

†Higher than primary school.

a Mediterranean diet positively affect mental health-related variables $^{(22,23)}$. Long-term effects of a low-fat, high-carbohydrate diet on quality of life were studied in the Canadian Diet and Breast Cancer Prevention Trial ${ }^{(11)}$. Participants showed a high score for self-reported mental and physical health after a median follow-up of $6 \cdot 8$ years. However, a similarly high score was observed in the control group, which reporting approximately $10 \%$ higher fat consumption. This result indicates that it may not be a single nutrient but rather the entire food pattern that affects quality of life. The effect of three different types of diets on quality of life were analysed in a sub-study of the Dietary Approaches to Stop Hypertension Trial ${ }^{(12)}$. The control diet of this trial represented typical food intakes of the American population. The fruit and vegetable diet was rich in fruit and vegetables but otherwise similar to the control diet. The third diet, called the combination diet, resembled a Mediterranean diet in several ways. Although participants' perception of quality of life improved with all dietary interventions, the greatest improvement was reported with the combination diet. Earlier epidemiological studies focused on the association of particular foods, food groups or nutrients with self-reported mental and physical health ${ }^{(7-10)}$. Higher fish, vegetable, fruit and dietary fibre consumption has been associated with better self-perceived health, particularly mental health ${ }^{(7-10)}$. It is worth noting that high consumption of these dietary components is part of the characteristics of the Mediterranean dietary pattern. The analysis of the impact of a single food or nutrient on health outcomes has its limitations ${ }^{(19)}$. Therefore, dietary patterns have gained considerable attention in the past two decades. Indeed, people do not consume nutrients or single foods but combinations of foods. Furthermore, dietary components may interact, making the search for associations between single dietary factors and health outcome more difficult.

The reported health effects of the Mediterranean diet have a solid biological foundation ${ }^{(1,2)}$. In addition, this diet is embedded in a social network that may contribute to increased personal well-being. Although speculative, it is reasonable to assume that a higher adherence to the Mediterranean diet also reflects a higher adherence to the Mediterranean lifestyle, including eating at home, expending time cooking, sharing lunchtime with other people and going to the market to buy foods. Indeed, using lunchtime for social communication and regeneration within family structures may improve selfperceived quality of life. It is known that social and family support can promote health by providing persons with positive experiences, socially rewarding roles or improved ability to 
Table 3. Lifestyle, anthropometric and socio-demographic variables according to tertile distribution of self-reported physical health (SF-12 Health Survey physical component score) in men and women (adjusted for age) ${ }^{*}$

(Mean values and $95 \% \mathrm{Cl}$ )

\begin{tabular}{|c|c|c|c|c|c|c|c|c|}
\hline & \multicolumn{4}{|c|}{ Men } & \multicolumn{4}{|c|}{ Women } \\
\hline & $\begin{array}{l}\text { First tertile } \\
(13.9-50.8)\end{array}$ & $\begin{array}{l}\text { Second tertile } \\
(50 \cdot 9-55 \cdot 3)\end{array}$ & $\begin{array}{l}\text { Third tertile } \\
(55 \cdot 4-67 \cdot 1)\end{array}$ & $\begin{array}{l}P \text { for linear } \\
\text { trend }\end{array}$ & $\begin{array}{l}\text { First tertile } \\
(14 \cdot 3-46 \cdot 1)\end{array}$ & $\begin{array}{l}\text { Second tertile } \\
(46 \cdot 2-54 \cdot 8)\end{array}$ & $\begin{array}{l}\text { Third tertile } \\
(54.9-68.4)\end{array}$ & $\begin{array}{l}P \text { for linear } \\
\text { trend }\end{array}$ \\
\hline$n$ & 1125 & 1186 & 1138 & & 1218 & 1221 & 1258 & \\
\hline Age (years) & & & & $<0.001$ & & & & $<0.001$ \\
\hline Mean & $57 \cdot 9$ & $54 \cdot 0$ & $51 \cdot 0$ & & $58 \cdot 0$ & 53.5 & $50 \cdot 0$ & \\
\hline $95 \% \mathrm{Cl}$ & $57 \cdot 2,58 \cdot 5$ & $53 \cdot 4,54 \cdot 6$ & $50 \cdot 3,51 \cdot 6$ & & $57 \cdot 4,58 \cdot 6$ & $52 \cdot 9,54 \cdot 1$ & $49 \cdot 4,50 \cdot 6$ & \\
\hline Current smokers & & & & 0.002 & & & & 0.987 \\
\hline$\%$ & 34.5 & 31.7 & $28 \cdot 3$ & & $16 \cdot 4$ & $17 \cdot 2$ & $16 \cdot 2$ & \\
\hline $95 \% \mathrm{Cl}$ & $31 \cdot 7-37 \cdot 2$ & $29 \cdot 1,34 \cdot 3$ & $25 \cdot 6,31 \cdot 1$ & & $14 \cdot 4,18 \cdot 5$ & $15 \cdot 2,19 \cdot 2$ & $14 \cdot 2,18 \cdot 2$ & \\
\hline Alcohol consumption (g/d) & & & & 0.900 & & & & $<0.001$ \\
\hline Mean & $15 \cdot 6$ & $16 \cdot 3$ & $15 \cdot 7$ & & 3.9 & 4.7 & 5.0 & \\
\hline $95 \% \mathrm{Cl}$ & $14 \cdot 5,16 \cdot 7$ & $15 \cdot 3,17 \cdot 4$ & $14 \cdot 6,16 \cdot 7$ & & $3.4,4 \cdot 3$ & $4 \cdot 3,5 \cdot 2$ & $4 \cdot 6,5.5$ & \\
\hline LTPA $($ MET $\times$ min/d) & & & & 0.006 & & & & $<0.001$ \\
\hline Mean & 350 & 365 & 396 & & 202 & 244 & 283 & \\
\hline $95 \% \mathrm{Cl}$ & 328,373 & 344,386 & 373,418 & & 188,216 & 230,258 & 269,296 & \\
\hline BMI $\left(\mathrm{kg} / \mathrm{m}^{2}\right)$ & & & & $<0.001$ & & & & $<0.001$ \\
\hline Mean & 28.4 & 27.9 & $27 \cdot 3$ & & $28 \cdot 7$ & $27 \cdot 0$ & $25 \cdot 8$ & \\
\hline $95 \% \mathrm{Cl}$ & $28 \cdot 2,28 \cdot 6$ & $27 \cdot 7,28 \cdot 1$ & $27 \cdot 0,27 \cdot 5$ & & $28 \cdot 4,29 \cdot 0$ & $26 \cdot 7,27 \cdot 3$ & $25 \cdot 5,26 \cdot 0$ & \\
\hline Educational level $\dagger$ & & & & $<0.001$ & & & & $<0.001$ \\
\hline$\%$ & $41 \cdot 1$ & $47 \cdot 3$ & 57.5 & & $36 \cdot 2$ & $45 \cdot 3$ & 56.0 & \\
\hline $95 \% \mathrm{Cl}$ & $38 \cdot 2,43 \cdot 9$ & $44.5,50 \cdot 0$ & $54 \cdot 7,60 \cdot 3$ & & $33 \cdot 6,38 \cdot 8$ & $42 \cdot 8,47 \cdot 9$ & $53 \cdot 4,58 \cdot 6$ & \\
\hline $\begin{array}{l}\text { Drug treatment and/or } \\
\text { medical history of }\end{array}$ & & & & & & & & \\
\hline Dyslipidaemia & & & & 0.001 & & & & 0.051 \\
\hline$\%$ & $36 \cdot 6$ & 34.4 & $29 \cdot 7$ & & $32 \cdot 0$ & $30 \cdot 1$ & $28 \cdot 3$ & \\
\hline $95 \% \mathrm{Cl}$ & $33 \cdot 8,39.4$ & $31 \cdot 7,37 \cdot 1$ & $26 \cdot 9,32 \cdot 5$ & & $29 \cdot 4,34 \cdot 6$ & $27 \cdot 6,32 \cdot 6$ & $25 \cdot 8,30 \cdot 8$ & \\
\hline Diabetes & & & & $<0.001$ & & & & $<0.001$ \\
\hline$\%$ & $17 \cdot 0$ & $13 \cdot 2$ & $10 \cdot 2$ & & 14.4 & $10 \cdot 9$ & 8.84 & \\
\hline $95 \% \mathrm{Cl}$ & $15 \cdot 1,19 \cdot 0$ & $11 \cdot 3,15 \cdot 1$ & $8 \cdot 2,12 \cdot 1$ & & $12 \cdot 6,16 \cdot 2$ & $9 \cdot 1,12 \cdot 7$ & $7 \cdot 0,10 \cdot 6$ & \\
\hline Hypertension & & & & 0.001 & & & & \\
\hline$\%$ & $35 \cdot 6$ & 31.8 & $29 \cdot 0$ & & 33.8 & $29 \cdot 7$ & 23.8 & $<0.001$ \\
\hline $95 \% \mathrm{Cl}$ & $32 \cdot 9,38 \cdot 3$ & $29 \cdot 3,34 \cdot 4$ & $26 \cdot 3,31 \cdot 6$ & & $31 \cdot 4,36 \cdot 3$ & $27 \cdot 2,32 \cdot 0$ & $21 \cdot 4,26 \cdot 2$ & \\
\hline
\end{tabular}

LTPA, leisure-time physical activity; MET, metabolic equivalents.

${ }^{*}$ For details of subjects and procedures, see Methods.

†Higher than primary school.

cope with stressful events ${ }^{(27)}$. On the other hand, a recent report from the Centers for Disease Control and Prevention suggested an association between perceived social support and HRQL, at least in older people ${ }^{(28)}$.
Although HRQL is a measure of perceived health rather than a biological measure, self-related health status has been shown to be a powerful predictor of mortality at long term ${ }^{(4)}$.

Table 4. Regression coefficients and $95 \% \mathrm{Cl}$ of the association of the Mediterranean diet score with self-reported mental (SF-12 Health Survey mental component score (SF12 MCS)) and physical (SF-12 Health Survey physical component score (SF12 PCS)) health in men and women

\begin{tabular}{|c|c|c|c|c|c|c|}
\hline & \multicolumn{3}{|c|}{ SF12 MCS } & \multicolumn{3}{|c|}{ SF12 PCS } \\
\hline & Regression coefficient & $95 \% \mathrm{Cl}$ & $P$ & Regression coefficient & $95 \% \mathrm{Cl}$ & $P$ \\
\hline \multicolumn{7}{|c|}{ Men } \\
\hline \multicolumn{7}{|c|}{$\begin{array}{l}\text { Mediterranean } \\
\text { diet score (1 unit) }\end{array}$} \\
\hline Model $1^{*}$ & 0.149 & $0.047,0.257$ & 0.008 & 0.171 & $0.087,0.256$ & $<0.001$ \\
\hline Model $2 \dagger$ & 0.148 & $0.035,0.261$ & 0.010 & 0.160 & $0.076,0.246$ & $<0.001$ \\
\hline \multicolumn{7}{|c|}{$\begin{array}{l}\text { Women } \\
\text { Mediterranean } \\
\text { diet score (1 unit) }\end{array}$} \\
\hline Model 1 & 0.266 & $0.146,0.387$ & $<0.001$ & 0.220 & $0.123,0.318$ & $<0.001$ \\
\hline Model $2 \ddagger$ & 0.230 & $0.105,0.356$ & $<0.001$ & 0.068 & $-0.030,0.165$ & 0.176 \\
\hline
\end{tabular}


Previous explanations argued that perceived health reflects an individual's awareness of symptoms, diagnoses and performance decrements that are associated with mortality risk $^{(3)}$. In the same way, the British Heart Regional Study showed that men who reported poor health were more likely to report medication use, sickness absence, major illnesses, angina pectoris, breathlessness and other characteristics indicative of poorer health ${ }^{(4)}$.

It is important to note that, with the exception of self-perceived physical function in women, the associations between HRQL and adherence to the Mediterranean diet held after adjusting for cardiovascular risk factors, leisure-time physical activity and educational status. The present findings are in line with previous studies showing a worse cardiovascular risk profile and low levels of physical activity among men and women reporting low HRQL scores ${ }^{(29,30)}$. It is of interest to note that people affected by dyslipidaemia, hypertension or diabetes did not score negatively in perceived mental health.

Levels of self-reported physical functioning tended to be higher in both men and women with more than basic education; mental health was better in women with a higher educational level. The effect of socioeconomic class on HRQL may be a consequence of job insecurity and psychosocial and physical working conditions in men and material well-being at home and amount of household labour in women $^{(31)}$. On the other hand, greater health inequalities described previously in women in Spain could support the differences found in mental health outcomes in this group in the present study ${ }^{(32)}$.

The cross-sectional nature of the study design precludes drawing causal relationships. Furthermore, all dietary instruments such as FFQ measuring past food intake are vulnerable to random and systematic measurement errors. Finally, misreporting is an acknowledged source of measurement error in prospective or retrospective methods of dietary assessment using self-reported food intake records.

Since nutritional exposure has a paramount effect on health, it is not surprising that diet is a key public health concern. Although policy-makers have generated many public health efforts promoting various healthy diets for the general population, the impact of a healthy diet on self-perceived mental and physical health has not been analysed. The present study outcomes provide new evidence for the health benefits of the Mediterranean diet and should encourage public health policymakers to promote this dietary pattern.

In conclusion, self-perceived mental and physical health function was directly associated with adherence to the Mediterranean diet in both sexes. With the exception of physical health function in women, the magnitude and direction of these associations did not change, after adjusting for several confounders.

\section{Acknowledgements}

We appreciate the English revision made by Dr Elaine M. Lilly (Writers First Aid). The CIBEROBN and CIBERSP are initiatives from the Instituto de Salud Carlos III, Madrid, Spain. This research was supported by grant 2FD097-0297-CO2-01 from Fondo Europeo de Desarrollo Regional (FEDER) and by parts of grants from Spain's Ministerio de Sanidad y Consumo,
Instituto de Salud Carlos III, Red HERACLES RD06/0009, and Fondo de Investigación Sanitaria ISCIII CP 03/00115. M.-A. M. led data analysis and writing of the manuscript. H. S. and M. F. participated in the discussion and interpretation of results, and writing of the manuscript. J. M. and M.-I. C. provided their expertise in data analysis and in interpretation and discussion of results. They made substantial suggestions on the manuscript. The authors declare no conflict of interest.

\section{References}

1. Serra-Majem L, Roman B \& Estruch R (2006) Scientific evidence of interventions using the Mediterranean diet: a systematic review. Nutr Rev 64, S27-S47.

2. Schroder H (2007) Protective mechanisms of the Mediterranean diet in obesity and type 2 diabetes. J Nutr Biochem 18, 149-160.

3. Kaplan G, Goldberg D, Everson S, et al. (1996) Perceived health status and morbidity and mortality: evidence from the Kuopio ischaemic heart disease risk factor study. Int $J$ Epidemiol 25, 259-265.

4. Wannamethee G \& Shaper AG (1991) Self-assessment of health status and mortality in middle-aged British men. Int J Epidemiol 20, 239-245.

5. Trichopoulou A \& Lagiou P (1997) Healthy traditional Mediterranean diet: an expression of culture, history, and lifestyle. Nutr Rev 55, 383-389.

6. Molarius A, Berglund K, Eriksson C, et al. (2007) Socioeconomic conditions, lifestyle factors, and self-rated health among men and women in Sweden. Eur J Public Health 17, $125-133$.

7. Silvers KM \& Scott KM (2002) Fish consumption and self reported physical and mental health status. Public Health Nutr 5, 427-431.

8. Smith AP (2005) The concept of wellbeing: relevance to nutrition research. Br J Nutr 93, Suppl. 1, S1-S5.

9. Myint PK, Welch AA, Bingham SA, et al. (2006) Fruit and vegetable consumption and self-reported functional health in men and women in the European Prospective Investigation into Cancer-Norfolk (EPIC-Norfolk): a population-based crosssectional study. Public Health Nutr 10, 34-41.

10. Blank L, Grimsley M, Goyder E, et al. (2007) Communitybased lifestyle interventions: changing behaviour and improving health. $J$ Public Health 29, 236-245.

11. Hislop TG, Bajdik CD, Balneaves LG, et al. (2006) Physical and emotional health effects and social consequences after participation in a low-fat, high-carbohydrate dietary trial for more than 5 years. J Clin Oncol 24, 2311-2317.

12. Plaisted CS, Lin PH, Ard JD, et al. (1999) The effects of dietary patterns on quality of life: a substudy of the Dietary Approaches to Stop Hypertension trial. J Am Diet Assoc 99, S84-S89.

13. Ware JE, Kosinski M \& Keller A (1996) A 12-Item Short-Form Health Survey: construction of scales and preliminary tests of reliability and validity. Med Care 34, 220-233.

14. Gandek B, Ware J, Aaronson N, et al. (1998) Cross-validation of item selection and scoring for the SF-12 health survey in nine countries: results from the IQOLA Project. J Clin Epidemiol 51, 1171-1178.

15. Schroder H, Covas MI, Marrugat J, et al. (2001) Use of a three-day estimated food record, a 72-hour recall and a food-frequency questionnaire for dietary assessment in a Mediterranean Spanish population. Clin Nutr 20, 429-437.

16. Trichopoulou A, Kouris-Blazos A, Wahlqvist ML, et al. (1995) Diet and overall survival in elderly people. $\mathrm{Br}$ Med $J$ 311, $1457-1460$. 
17. Trichopoulou A, Costacou T, Bamia C, et al. (2003) Adherence to a Mediterranean diet and survival in a Greek population. $N$ Engl J Med 348, 2599-2608.

18. Martinez-Gonzalez MA, Fernandez-Jarne E, Serrano-Martinez M, et al. (2002) Mediterranean diet and reduction in the risk of a first acute myocardial infarction. An operational healthy dietary score. Eur J Nutr 41, 153-160.

19. Willett WC (1998) Nutritional Epidemiology. Oxford: Oxford University Press.

20. Elosua R, Garcia M, Aguilar A, et al. (2000) Validation of the Minnesota leisure time physical activity questionnaire in Spanish women. Investigators of the MARATDON Group. Med Sci Sports Exerc 32, 1431-1437.

21. Elosua R, Marrugat J, Molina L, et al. (1994) Validation of the Minnesota leisure time physical activity questionnaire in Spanish men. The MARATHOM Investigators. Am J Epidemiol 139, 1197-1209.

22. Halyburton AK, Brinkworth GD, Wilson CJ, et al. (2007) Low- and high-carbohydrate weight-loss diets have similar effects on mood but not cognitive performance. Am J Clin Nutr 86, 580-587.

23. Wells AS, Read NW, Laugharne JD, et al. (1998) Alterations in mood after changing to a low-fat diet. Br J Nutr 79, $23-30$.

24. Wardle J, Rogers P, Judd P, et al. (2000) Randomized trial of the effects of cholesterol-lowering dietary treatment on psychological function. Am J Med 108, 547-553.
25. Bowen DJ, Kestin M, McTiernan A, et al. (1995) Effects of dietary fat intervention on mental health in women. Cancer Epidemiol Biomarkers Prev 4, 555-559.

26. Weidner G, Connor SL, Hollis JF, et al. (1992) Improvements in hostility and depression in relation to dietary change and cholesterol lowering. The Family Heart Study. Ann Intern Med 117, 820-823.

27. Seeman TE (2000) Health promoting effects of friends and family on health outcomes in older adults. Am $J$ Health Promot 14, 362-370.

28. Centers for Disease Control and Prevention (2008) Social support and health-related quality of life among older adults. http://www. cdc.gov/mmwR/preview/mmwrhtml/mm5417a4.htm (accessed 14 February 2008).

29. Spertus J, Jones P, McDonell M, et al. (2002) Health status predicts long-term outcome in outpatients with coronary disease. Circulation 106, 43-49.

30. Bize R, Johnson J \& Plotnikoff R (2007) Physical activity level and health-related quality of life in the general adult population: a systematic review. Prev Med 45, 401-415.

31. Borrell C, Muntaner C, Benach J, et al. (2004) Social class and self-reported health status among men and women: what is the role of work organization, household material standards and household labour? Soc Sci Med 58, 1869-1887.

32. Regidor E, Gutierrez-Fisach J, Dominguez V, et al. (2002) Comparing social inequalities in health in Spain: 1987 and 1995/97. Soc Sci Med 54, 1323-1332. 